\title{
MONDO: A neutron tracker for particle therapy secondary emission fluxes measurements
}

\author{
R. Mirabelli $\left({ }^{1}\right)\left({ }^{2}\right)\left({ }^{*}\right)$ \\ $\left.{ }^{1}\right)$ INFN, Sezione di Roma - Roma, Italy \\ $\left(^{2}\right)$ Dipartimento di Fisica, Sapienza Università di Roma - Roma, Italy
}

received 15 February 2017

\begin{abstract}
Summary. - A charged particle passing through matter releases a considerable amount of energy at the end of its path. Thus, thanks to the spatial distribution of the deposited energy, particle therapy allows treating tumors with greater accuracy and efficiency than conventional radiotherapy. However, during the treatments, several secondary particles are produced from the interactions between therapeutic beams and human tissues and contribute to the total dose delivered to the patient. Since neutrons can release a significant dose far away from the tumour region, a precise measurement of their flux, production energy and angle distribution is eagerly needed to improve the treatment planning system and to estimate the normal tissue toxicity in the target region and establish if/where there could be the risk of secondary neoplasms. The MONDO (MOnitor for Neutron Dose in hadrOntherapy) project aims at detecting secondary neutrons with high efficiency and good backtracking precision.
\end{abstract}

\section{1. - Introduction}

The main advantage of Charged Particle Therapy (CPT), compared to conventional radiation therapy, is that the dose released is concentrated in the tumour region, thus sparing healthy surrounding tissues. In fact, protons and light ions release the maximum dose at the end of their path in the well-known Bragg peak. The Bragg peak position and width depend on the beam energy and composition. On the other hand, the production of secondary particles due to the fragmentation of the beam inside the patient is one of the principal complication in CPT. The secondary radiation component has to be precisely characterized because it can be used for online beam monitoring and it contributes to the effective dose delivered to the patient during the treatment [1]. However, the neutron component is still affected by large experimental uncertainties and is almost,

$\left(^{*}\right)$ E-mail: riccardo.mirabelli@roma1.infn.it 


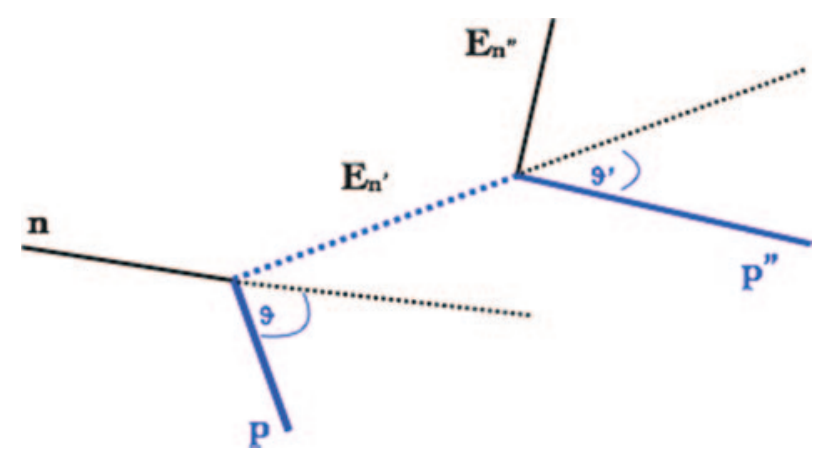

Fig. 1. - Schematic representation of a neutron interacting with double elastic scattering. From the reconstruction of the proton tracks and the measurement of their kinetic energy and production angles, the event can be fully reconstructed and direction and energy of the incident neutron can be computed.

yet, unexplored [2]. Neutrons, because of their short attenuation length, represent the most abundant and harmful radiation exiting from the patient body and contributing to a substantial dose deposition in tissues that are not directly targeted or crossed by the beam. A deep knowledge of the secondary neutron production is crucial because the risk of developing a radiogenic second malignant neoplasm (SMN), years or decades after undergoing a treatment, is one of the main concerns in CPT administration and planning [3]. A complete characterization of the neutron production and the related dose deposition is crucial to provide a better treatment plan to patients, maximizing the therapy effectiveness while reducing secondary effects. The aim of the MONDO project (MOnitor for Neutron Dose in hadrOntherapy) [4] is to characterise the secondary neutron production in CPT to improve Monte Carlo (MC) and analytical models on which the treatment planning system (TPS) relies building a tracker for the measurement of neutrons in the (20-400) MeV range.

\section{2. - MONDO project}

$2 \cdot 1$. Detection strategy. - The principal interactions of fast and ultrafast neutrons with the plastic scintillators are elastic scattering with hydrogen nuclei (n-p) and carbon nuclei (n-C). The elastic scattering kinematics links neutron and proton momenta, and the $n-p$ events can be used for the neutron detection.

The incident neutron energy can be obtained using the recoiling proton energy when the neutron direction is known, according to the equation

$$
E_{n}=E_{p} / \cos ^{2} \theta
$$

where $E_{n}$ and $E_{p}$ are kinetic energies, respectively, for the neutron and recoil proton and $\theta$ is the angle between them (fig. 1).

When both neutron energy and direction are unknown, neutrons undergoing double elastic scattering interactions can be used for the complete reconstruction of the event. In this case, the tracking and energy resolution achievable in the detection and reconstruction of the two recoiling protons will drive the final neutron energy and angular resolutions. 


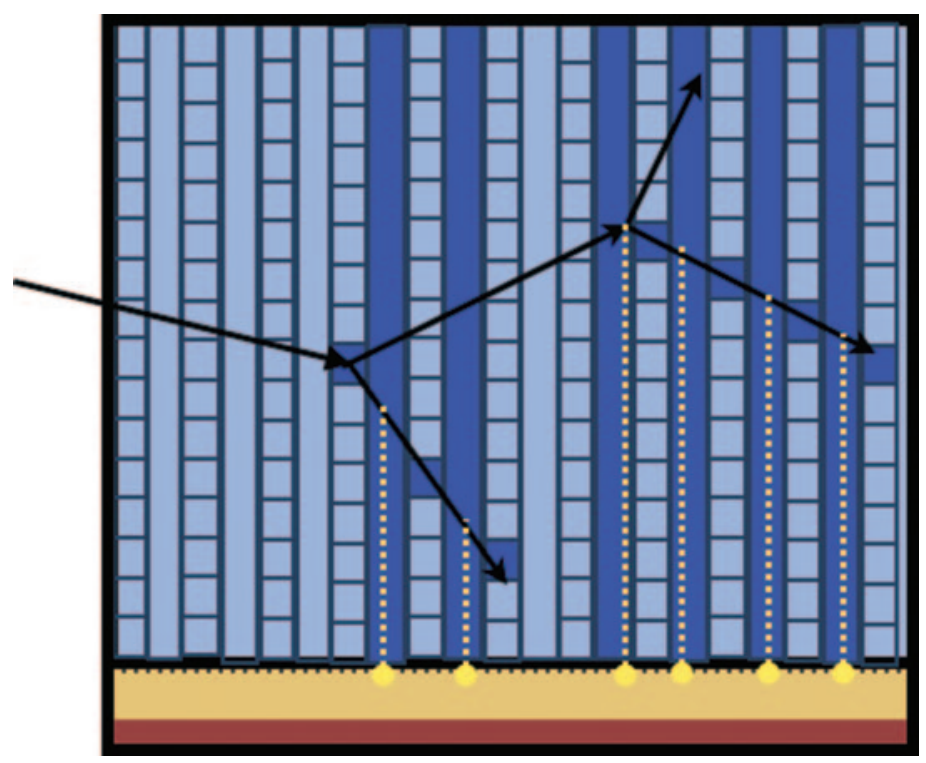

Fig. 2. - Design of the MONDO tracker. The fibres activated by the passage of the scattered protons are highlighted.

2*2. The neutron tracker. - The MONDO neutron tracker (fig. 2) is composed by subsequent orthogonal layers of $250 \mu \mathrm{m}$ plastic fibres, that act both as target and as active component of the detector. The final size of the object was defined considering the average neutron interaction length in plastic ( $\sim 10 \mathrm{~cm}$ for $20-300 \mathrm{MeV}$ energy range). The final tracker dimensions $(10 \times 10 \times 20 \mathrm{~cm})$ were decided considering also the cost of the materials and the high efficiency requested. The size of the fibres was chosen to have enough tracking points along the orthogonal views to track recoiling protons with kinetic energy of at least $15 \mathrm{Mev}$. In this scenario, the range of protons in plastic is $\sim 1.5 \mathrm{~mm}$ and a squared fibre of $250 \mu \mathrm{m}$ allows tracking particles with at least three hits in both views.

$2 * 3$. Detector readout. - The technology used for the readout system of the MONDO tracker is based on CMOS-based Single Photon Avalanche Diode arrays (SPAD) designed by Fondazione Bruno Kessler $(\mathrm{FBK})\left({ }^{1}\right)$. An optical readout based on a Triple-GEM detector was also studied and tested $[5,6]$. The design of an optimized sensor (SBAM) capable of matching the specific MONDO readout has been developed from the analysis reported of the performance of spadnet, a SPAD-based prototype, and optimized for PET analysis with LYSO crystals [7]. The spadnet prototype was built out of a SPAD matrix of $16 \times 8$ squared pixels of $600 \mu \mathrm{m}$ side that implements a self triggering logic. Each pixel contains 720 SPADs which can be individually switched off, to reduce the impact of noisy channels on the detector sensitivity. The implemented self triggering logic is synchronous with a global clock (running at up to $100 \mathrm{MHz}$ ) and requires that the total number of SPADs triggering within a clock bin is greater than the threshold set by the user. After a proper calibration, the expected extremely low dark counts of

$\left({ }^{1}\right)$ wWW. fbk.eu 

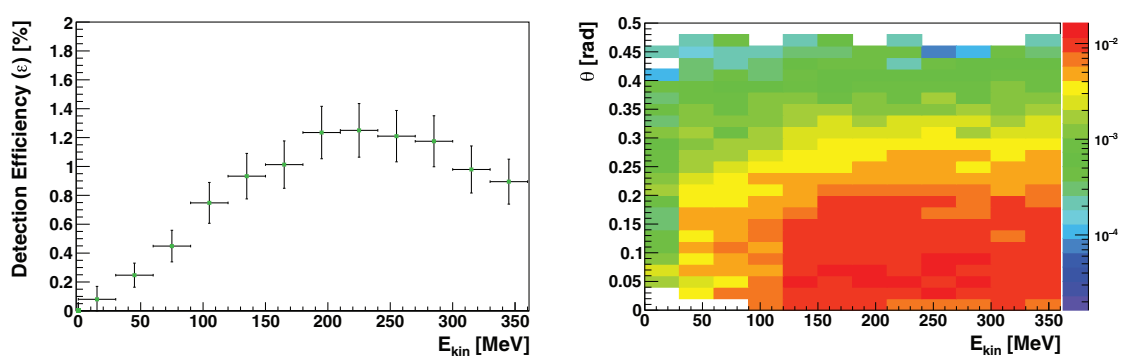

Fig. 3. - Left: detection efficiency (indeed as the capability of completely contain two subsequent recoiled proton trajectories) as a function of neutron kinetic energy. Right: efficiency, represented by the color palette, as a function of neutron kinetic energy and emission angle.

the sensor spadnet will allow to acquire data using the self-triggering strategy even in the case of the low signals expected from the MONDO tracker. The sensor development is subject to the characterization of the specific signals produced by charged particles impinging on the matrix of scintillating fibres.

\section{3. - Simulation}

A preliminary Monte Carlo simulation of the detector was developed using FLUKA [8] to optimize the detector layout and to study its efficiency [9]. The energy released in the fibres by secondary protons emitted from neutron elastic scattering has been studied. Neutrons generated were isotropically emitted in a $5^{\circ}$ aperture cone with axis coincident with the detector central axis and in the energy range from 20 up to $400 \mathrm{MeV}$.

In a first conservative approach we evaluated the efficiency as the capability of completely containing two subsequent recoiled proton trajectories.

Figure 3 (left) shows the containment capacity as a function of neutron kinetic energy: the number of fibres crossed by protons increases with their energy and also the tracking efficiency increases as the incoming neutron energy rises. At high energy the efficiency reaches a plateau which is determined by the total detector size and consequently by the ability of fully containing two subsequent recoiling protons. The simulation, as is shown in fig. 3 (right), proved also that the containment efficiency is inversely proportional to the proton emission angle. This is due to geometrical reasons: given the detector transversal dimensions, the probability for a proton emitted at large angles to escape from the detector volume is greater, compromising the tracking efficiency.

\section{4. - Preliminary test on a detector prototype}

A measurement of the fibres light readout capability of the spadnet prototype has been carried out at the Beam Test Facility (BTF) of the INFN - Laboratori Nazionali di Frascati (LNF) with electrons with energies in the (100-450) MeV range. The aim of the test was the assessment of the sensor capabilities for the readout of the scintillation light produced within the fibres.

The measurements were performed using a single layer of fibres $250 \mu \mathrm{m}$ thick covering an area of $4 \times 4 \mathrm{~cm}^{2}$ (in the following referred to as demonstrator). The demonstrator, readout by the spadnet sensor, was placed in a vertical plane orthogonal to the electron beam direction, as shown in fig. 4 . The fibres used to built the plane $(\sim 160)$ were 

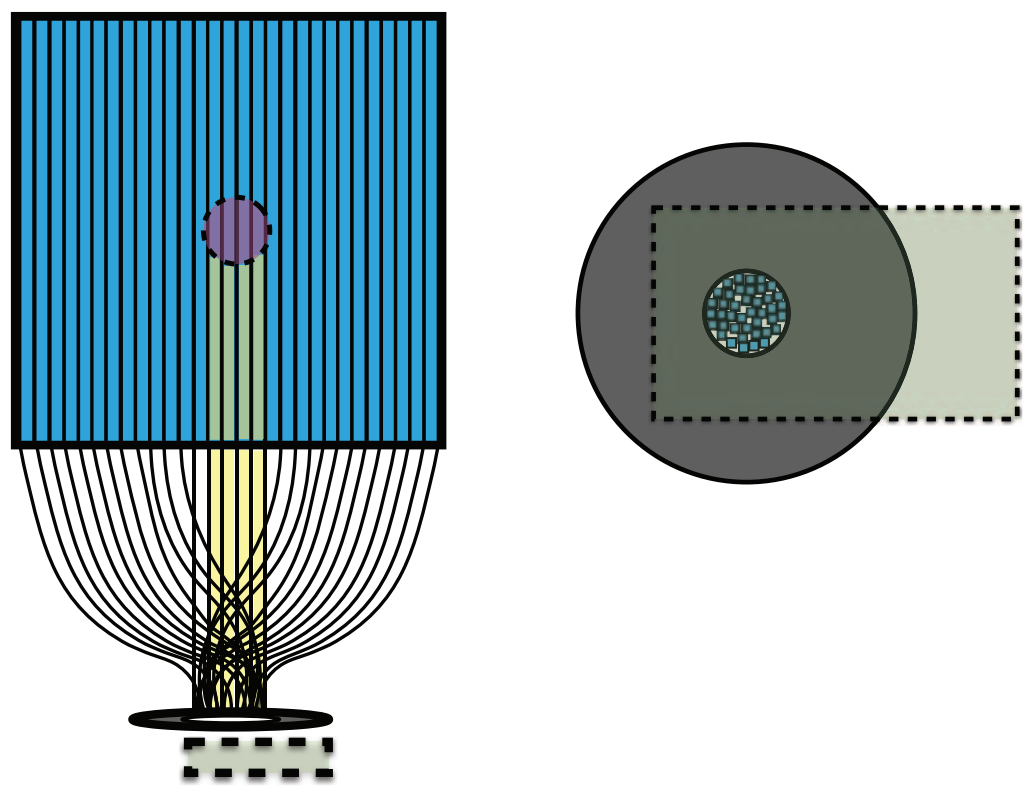

Fig. 4. - Left: single demonstrator layer, $4 \times 4 \mathrm{~cm}^{2}$ area, readout setup. The plane is held vertically, the central circle shows the sketch of the impinging electron beam in the perpendicular direction. the fibres that are producing light, due to the interactions with the beam, are highlighted. Right: the fibres are collected in a PVC support and are readout by the spadnet sensor.

grouped in a mesh, collected by a PVC support and connected to the readout sensor surface shown as a rectangle at the bottom of fig. 4 (left). Between the fibres and the readout pixels no mechanical matching was implemented: we are hence assuming, in the data analysis, that the fibres were randomly and homogeneously distributed in the sensor. Figure 4 (right) shows a view from the bottom, where both the PVC support holding the fibres and the readout sensor are visible. The beam multiplicity used for the test was carefully tuned in order to collect a significant data sample in which, in a given event, only one electron is expected to cross a single fibre.

4*1. Detector expected performances. - The detector expected performances can be evaluated once the number of produced photons in the fibres is known.

At $450 \mathrm{MeV}$, electrons act like a MIP (Minimum Ionizing Particle) and the mean energy released in a $250 \mu \mathrm{m}$ plastic fibre is $\sim 50 \mathrm{keV}[10]$. Considering the light yield and the trapping efficiency of a double-cladding fibre, the number of photoelectrons produced from a single event is $\sim 30$. In order to convert the number of photons in photoelectrons, the quantum efficiency of the photocatode and the fill factor of the sensor have been considered. The final number of expected photoelectrons is $\sim 3$.

A summary of the calculation is presented in table I.

A conservative assumption on the expected energy and direction angle resolution performances attainable with the MONDO tracker can be calculated using the published Sontrack results $[11,12]$, where $\sigma(E) / E(F W H M) \sim 4 \%$ and $\sigma(\theta) \sim 4.6^{\circ}$ (at $35 \mathrm{MeV}$ improving with energy) were measured. As the size of the MONDO tracker is larger, with a readout system that is expected to be as efficient as the commercial CCD based 
TABLE I. - Expected number of photons and photoelectrons in the readout system that are produced by an electron that cross a $250 \mu \mathrm{m}$ side fibre.

\begin{tabular}{rc}
\hline Deposit energy in fibre & $50 \mathrm{keV}$ in $250 \mu \mathrm{m}$ \\
\hline Fibre light yield & $8000 \gamma \mathrm{MeV}$ \\
Fibre trapping efficiency & $7 \%$ \\
$N_{\gamma}^{\text {prod }}$ & $\sim 30 \gamma$ \\
\hline & Sensor FBK \\
\hline Quantum Efficiency & $33 \%$ \\
Fill Factor & $40 \%$ \\
$N_{\text {SpadOn }}$ & $60 \%$ \\
\hline$N_{\text {ph.el }}$ & $\sim 3$ \\
\hline
\end{tabular}

one implemented within Sontrack, the above-mentioned performances can be considered as a conservative estimation.

4.2. Scintillation light measurement. - The number of detected photoelectrons in a given event corresponds to the number of SPAD that are activated in each pixel. In fig. 5 the number of detected photoelectrons in each pixel sensor is shown. The distribution was fitted with a Poisson function, superimposed to the figure. The obtained mean value $(\lambda)$ of the distribution is $2.99 \pm 0.15$, in agreement with the expected number of photoelectrons for single electrons crossing the plane.

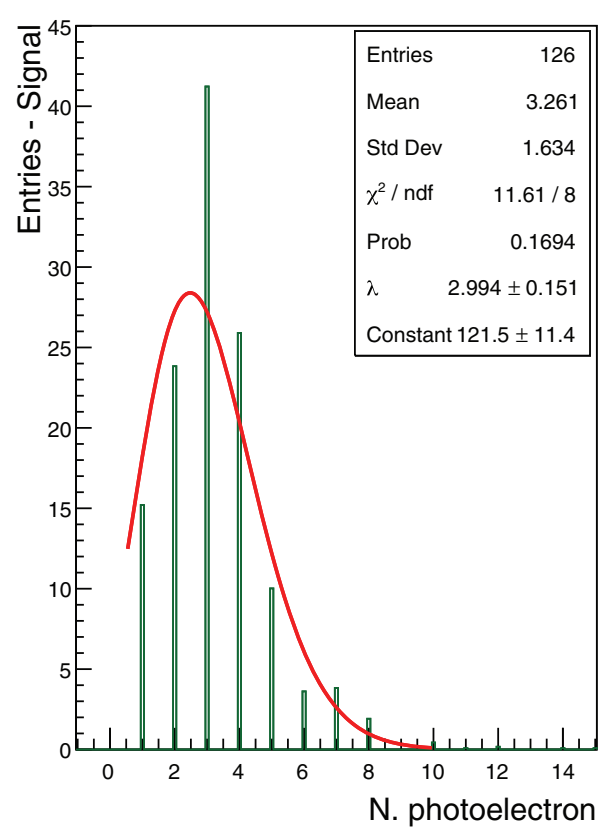

Fig. 5. - Distribution of the number of detected photoelectrons for each pixel of the spadnet sensor. The result of a fit performed using a Poisson distribution is superimposed. 


\section{5. - Conclusion}

The main goal of the MONDO project is to measure the neutron flux as a function of the energy and emission angle. This will allow to improve the knowledge of the secondary neutrons component produced during particle therapy treatments thus validating the models used for developing patients' TPS. The estimation of the total neutron dose contribution in regions far from the tumour volume is particularly important in paediatric cases. The measurement of this dose contribution will significantly help to understand and consequently to reduce unwanted secondary effects related to the therapy. The results shown in this review revealed that a fully digital CMOS approach is suitable for the MONDO tracker: the spadnet sensor is capable of detecting the light in the fibres even when small signals are produced by MIP impinging on the demonstrator. However, as the spadnet prototype pixels are too large with respect to the fibres and the rate of false events generated by the implemented discrimination logic is not negligible, the development of a new sensor optimized for the MONDO application is required. In particular, the new design performed in partnership with FBK will implement a smart discrimination mechanism (based on double level discriminator) specifically tailored for the proton tracks detection and will match the tracker geometry. The photons sensitivity per pixel will be improved and the pixel size will be optimized to maximize the readout effciency of the MONDO fibres (double cladding, squared, $250 \mu \mathrm{m}$ side).

$$
* * *
$$

I am grateful to Dr. Michela Marafini and the whole MONDO Collaboration for scientific support during the analysis process. I would like to thank FBK people for their work in the design of the sensor and Marco Magi (SBAI Department) for the construction of the mechanical structure of the detector. I also thank the BTF staff for beam-time and helpful support during the data taking. The project MONDO is supported by INFN Gruppo V with a Young Researchers Grant (2015-2016) and by SIR (Scientific Independence of young Researchers) MIUR Founding (2015-2018).

\section{REFERENCES}

[1] Faccini R. and Patera V., Mod. Phys. Lett. A, 30 (2015) 1540023.

[2] Hultqvist M. and Gudowska I., Phys. Med. Biol., 55 (2010) 6633.

[3] Newhauser W. and Durante M., Nat. Rev. Cancer, 11 (2011) 438.

[4] Marafini M. et al., Nucl. Instrum. Methods Phys. Res. A, 824 (2016) 210.

[5] Marafini M. et al., JiNST, 10 (2015) P12010.

[6] Marafini M. et al., Nucl. Instrum. Methods Phys. Res. A, 824 (2016) 562.

[7] Braga L.H.C. et al., IEEE J. Solid-State Circuits, 49 (2014) 301.

[8] Battistoni G. et al., Ann. Nucl. Energy, 82 (2015) 10.

[9] Valle S.M. et al., Nucl. Instrum. Methods Phys. Res. A, 845 (2016) 556.

[10] Marafini M. et al., Phys. Med. Biol., 62 (2017) 3299.

[11] Ryan J.M. et al., in Nuclear Science Symposium and Medical Imaging Conference, Vol. 1 (IEEE) 1999, p. 483.

[12] Donzella A. et al., Nucl. Instrum. Methods Phys. Res. A, 631 (2010) 58. 